\title{
Ökonomisierung der Medien aus systemtheoretischer Perspektive
}

\author{
Gabriele Siegert
}

Aus systemtheoretischer Sicht basiert eine Analyse der Ökonomisierung der Medien auf den Systemrationalitäten „Publizität" und „Geld“. Unter Rückgriff auf die Unterscheidung zwischen operativer Selbst- und Kontextsteuerung kann eine Ökonomisierung mit der besonderen Eignung von Geld als Medium der Kontextstewerung erklärt werden. Auf der Ebene der Medienorganisationen lässt sich durch die Verbindung mit der Theorie rationalen Handelns die Durchsetzungskraft der beiden Rationalitäten diskutieren. Interaktionen und Konvertierungsprozesse zwischen den beiden Systemen belegen nicht nur deren intensive Beziehungen zueinander, sondern sind auch Grundlage für Interpenetration. Interpenetrationszonen sind jene Bereiche, in denen die wechselseitige Anpassung an die je andere Operationslogik offensichtlich wird. Sie finden sich auf der Inhalteebene mit PR und Werbung, in der Funktionslogik der Online-Ökonomie und in der immensen Entwicklung der Organisations- und Unternehmenskommunikation. Daber lässt sich sowobl eine Ökonomisierung der Medien als auch eine Mediatisierung der Ökonomie feststellen.

Die Systemtheorie spielt in medienökonomischen Debatten bislang eine eher untergeordnete Rolle. Sie kann jedoch an die anderen medienökonomischen Theorie-Perspektiven sowohl auf der gesellschaftlichen Makroebene als auch auf der organisatorischen Mikroebene wichtige Anknüpfungspunkte aufweisen, an Theorien der Medienorganisation/-unternehmung über die Organisationssoziologie und -kommunikation, an die neoklassische Perspektive, denn der von Adam Smith als „invisible hand“ bezeichnete Marktmechanismus zielt auf eine systemische Logik, und sogar an den historischen Materialismus, denn systemtheoretische Konzeptionen fokussieren auf emergente Eigenschaften von Systemen und analysieren abstrakte Phänomene, wie z. B. den Markt oder das Kapital (vgl. auch Willke, 1996: 191ff).

Um aufzuzeigen, welchen Beitrag die Systemtheorie zur Ökonomisierungsdebatte leisten kann, müssen Funktion, Leistung und Logik der Systeme Medien und Ökonomie geklärt und die potenziellen, wechselseitigen Beziehungen zwischen den Systemen diskutiert werden. Zugleich sind die Möglichkeiten einer Überlagerung der beiden Rationalitäten und der Vorherrschaft der ökonomischen Systemlogik theoretisch zu erörtern. Ein systemtheoretisch orientierter, empirischer Nachweis der Ökonomisierung der Medien kann an dieser Stelle nicht erbracht werden. Die verwendeten Beispiele sind deshalb als argumentative Illustrationen gedacht. ${ }^{1}$

1 Die empirische Umsetzung systemtheoretischer Konzeptionen ist von vielen Schwierigkeiten gekennzeichnet. Gleichwohl belegt dies nicht nur die vielkritisierte „Empiriefeindlichkeit“ der Systemtheorie, sondern auch die Empirieproblematik von Makroanalysen und Theorien höherer Komplexität. 


\section{Systemtheoretische Grundlagen}

Systeme werden anhand der System-Umwelt-Differenz gebildet, die darauf basiert, dass Systeme mittels systemeigener Steuerungsmedien (Codes) kommunizieren. Dass Systeme sowohl sinnkonstituiert als auch sinnkonstituierend sind, verdeutlicht, dass die auf einem spezifischen Sinn aufbauende systeminterne Kommunikation von der anderer Systeme unterschieden werden kann. Jedes System folgt also seiner Eigenlogik bzw. seiner eigenen Rationalität. Insofern beobachtet auch jedes Teilsystem das gesellschaftliche Gesamtsystem und andere Teilsysteme in erster Linie im Rahmen seiner eigenen Rationalität.

Die Abgrenzung des Mediensystems schließt an die Konzeption von Marcinkowski (1993) an. Er sieht die besondere Leistung des Systems Publizistik in der „Ausstattung von Themen mit Publizität“, womit es deren Kommunikationserfolg wahrscheinlicher macht, also Aufmerksamkeit generiert. Die dazugehörende System-Umwelt-Differenz liegt in der Unterscheidung „öffentlich - nicht öffentlich“. Dabei stellt er der spezifischen Themen-Gebundenheit anderer subsystemischer Kommunikation die prinzipielle Themen-Offenheit des publizistischen Systems gegenüber. Die Primärfunktion des Mediensystems für die Gesamtgesellschaft ist es, durch Verarbeitung von Umweltkomplexität deren Selbstbeobachtung zu ermöglichen und eine Selbstbeschreibung der Gesellschaft herzustellen. Trotz prinzipieller Themen-Offenheit orientiert es sich an seiner Veröffentlichungsrationalität, wobei Nachrichtenwerte als Sekundärcodes oder Programme systemintern Wissen und Erfahrung in Selektionen der Umweltwahrnehmung konkretisieren können (vgl. u. a. Gerhards, 1994: 89; Westerbarkey, 1995: 154ff.). Die Tatsache, dass Medien sowohl ein autonomes soziales Subsystem als auch ein Metasystem zur Vermittlung zwischen allen anderen Subsystemen sind, verleiht ihnen einen grundsätzlichen Doppelcharakter (vgl. Westerbarkey, 1995: 154).

Die Primärfunktion des geschichtlich sehr früh ausdifferenzierten Wirtschaftssystems (vgl. dazu ausführlich Luhmann 1996b) liegt in der „Vorsorge für die Befriedigung zukünftiger Bedürfnisse“. Der kommunikative Code ist Geld. Dabei ist Geld in hohem Maße selektiv, weil es die Ausklammerung von nicht monetarisierbaren Relevanzen erfordert, d. h. „daß alles, was überhaupt auf wirtschaftliche Verwendung hin angesehen wird, auf einen Geldausdruck reduziert wird“ (Luhmann, 1996b: 238). Zahlungen als Letztelemente des Systems, als zugrundeliegende kommunikative Handlungen, koppeln Selbstreferenz - als Verbindung zum Code Geld - und Fremdreferenz - als Verbindung zu systemfremden Gütern und Dienstleistungen. Über Preise wird eine Information über Zahlungserwartungen, also eine Beobachtung von Beobachtung, ermöglicht.

Mit den beiden Systemen „Medien“ und „Ökonomie“ treffen auch zwei unterschiedliche Rationalitäten aufeinander: die Veröffentlichungsrationalität des Mediensystems und die Geldrationalität des Wirtschaftssystems. Da ein System sich aus den kommunikativen Handlungen, die seiner eigenen Rationalität entsprechen, zusammensetzt und die gesellschaftlichen Teilsysteme aufgrund ihrer spezifischen Problemlösungsfunktion gleichrangig sind, kann es in dieser Perspektive der Systemtheorie Luhmannscher Prägung keine Ökonomisierung des Mediensystems geben (vgl. auch Theis-Berglmair, 2000: 311). Analysiert werden kann aber, wie die beiden Systeme ihren wechselseitigen Leistungsaustausch organisieren. Für einen weiterreichenden Erklärungsbeitrag zur Ökonomisierungsdebatte kann auf folgende systemtheoretische Konzepte zurückgegriffen werden:

1. Die Verknüpfung unterschiedlicher Systemrationalitäten kann mit der Unterscheidung von operativer Steuerung als Selbststeuerung der Systeme und kontextueller 
Steuerung als dem Setzen von Bedingungen erklärt werden. Durch die Kontextsteuerung wird also mehr als nur eine Rationalität für das jeweilige Teilsystem relevant, dessen Autonomie durch die Selbststeuerung jedoch im Grundsatz erhalten bleibt. Ein Erklärungsansatz für die Dominanz ökonomischer Rationalität findet sich in der besonderen Eignung von Geld als Mittel zur kontextuellen Konditionierung von selbststeuernden Systemen. Geld hat die besondere Eigenschaft, „über die Grenzen der eigentlichen Ökonomie hinauszuwirken und Transaktionen jeglicher Art mit dem Virus des ökonomischen Kalküls zu infizieren." (Willke, 1998: 186)

2. Medienökonomisch erscheinen vor allem solche System-Konzeptionen erklärungskräftig, die akteurstheoretische Rekonstruktionen und Anschlüsse bieten und damit auf Handlungen - und nicht ausschließlich wie Luhmann (1988: 192) auf Kommunikationen - als Letztelemente von Systemen rekurrieren (vgl. u.a. Schmidt, 1996: 28; Weischenberg, 1994: 430). Da die Verantwortlichkeit der Medienorganisationen für das mediale Angebot, für die Berücksichtigung verschiedenster Interessen sowie für Vielfalt und Qualität von Medieninhalten medienökonomisch eine wichtige Rolle spielt, müssen analytisch a) Handlungen und Strategien ausgemacht werden können und b) auf Akteure rückführbar sein. Durch die Verknüpfung der Systemtheorie mit der Theorie rationalen Handelns (vgl. Schimank, 1985 und 1988) können Medienorganisationen als kollektive Akteure begriffen werden. Sie können unter systemisch gesetzten, strukturellen Restriktionen dennoch auch ihre eigenen, spezifischen Ziele verfolgen. D. h. Systeme als situationsübergreifende, generalisierte Handlungsorientierungen konditionieren die Auswahlmöglichkeiten der Akteure, lassen aber Handlungsspielraum (vgl. Gerhards, 1994: 80f.).

3. Im Konzept der Interpenetration wird die wechselseitige Durchdringung von Systemen und die entsprechende Vernetzung von Systemlogiken behandelt (vgl. Münch, 1991: 135ff. und 332ff.). Trotz relativer Autonomie der einzelnen Teilsysteme werden normative Widersprüche, faktische Konflikte, strukturelle Inklusionen, aber auch unterschiedliche Machtverhältnisse bearbeitbar (vgl. für die Beziehung Medien und Politik: Westerbarkey, 1995: 154). Auch hier steht die Organisation des gegenseitigen Leistungsaustausches, der durch die jeweiligen Codes gesteuert wird, im Mittelpunkt. Mit der Einführung von Institutionen zur Konvertierung der Codes kann der wechselseitigen Instrumentalisierung theoretisch begegnet werden.

\section{2. Ökonomisierung der Medienindustrie}

Vorausgehend muss darauf hingewiesen werden, dass der Begriff „Mediensystem“ in medienökonomischen Diskursen meistens in seiner alltäglichen Bedeutung als Medienbranche und nicht unter Einbeziehung der Systemtheorie verwendet wird. Dies ist deshalb explizit zu bedenken, weil die Handlungen der Medienbranche, abhängig davon, welche situationsübergreifende, generelle Handlungsorientierung vorherrscht, dem ökonomischen System, dem Mediensystem, dem Erziehungssystem, dem politischen System, oder anderen Systemen angehören.

Eine Ökonomisierung der Medienindustrie könnte danach nachgewiesen werden, wenn alle zur Medienindustrie gehörenden kommunikativen Handlungen - sowohl die individueller als auch die korporativer Akteure - differenziert, aufgelistet und den entsprechenden Codes und Systemen zugeordnet werden können und dann eine überproportionale Zuordnungshäufigkeit zum Wirtschaftssystem festgestellt werden würde. Ist dabei der Anteil der an der Veröffentlichungsrationalität des Mediensystems orientierten Handlungen gering, muss die Frage gestellt werden, ob überhaupt genügend Eigen- 
komplexität und Kapazitäten zur gesellschaftlichen Selbstbeobachtung vorhanden sind, und somit die Komplexität der zu beobachtenden Umwelt verarbeitet, ob also von einem ausdifferenzierten Mediensystem gesprochen werden kann. Hier sei jedoch auf verschiedene Ausführungen verwiesen, die die Ausdifferenzierung eines eigenständigen Mediensystems nachweisen. ${ }^{2}$

Dennoch ist die mit Zahlungen verknüpfte Vermarktung aller dafür geeigneten Veröffentlichungsakte und Angebote sowie die Orientierung der medialen Produktion an Zahlungen ein wesentlicher Indikator dafür, dass die ökonomische Rationalität Eingang in das Mediensystem gefunden hat. Dies soll im Folgenden unter Rückgriff auf die skizzierten systemtheoretischen Ansätze auf drei Ebenen diskutiert werden. ${ }^{3}$

\subsection{Selbststenerndes Mediensystem und Kontextstenerung durch Geld}

Folgende Beispiele sollen die Ökonomisierungsthese illustrieren und zeigen, dass Geld als Handlungsorientierung für Veröffentlichungsakte an Bedeutung gewinnt: Die Etablierung des privaten Rundfunks war durch die Ausrichtung der Medienunternehmen auch auf Gewinnmaximierung eindeutig mit der Zunahme von ökonomischen Handlungen verbunden. Aktuell nehmen ökonomische Akte quantitativ und qualitativ durch den Börsengang von Medienunternehmen zu, der eine zweite Ebene ökonomischer Orientierung in die Unternehmensführung einführt. Zugleich findet sich eine Ökonomisierung der Ökonomisierung (vgl. Altmeppen, 2001) durch die wechselseitige Orientierung der Medienunternehmen an den ökonomischen Handlungen und Strategien ihrer Wettbewerber. Die Einführung und Etablierung individueller Abrechnungssysteme im digitalen Rundfunk und in der Online-Kommunikation verstärken diesen Trend (vgl. dazu auch Bruns/Marcinkowski/Nieland/Ruhrmann/Schierl, 1996: 32ff.), weil sie ehemals nur an der Veröffentlichungsrationalität orientierte Handlungen mit Zahlungen verknüpfen.

Unterscheidet man die operative Steuerung von Systemen, die immer nur das zu steuernde System selbst ausführen kann, und die Kontextsteuerung, die nicht in die interne Operationsweise des Systems eingreift, sondern Bedingungen zur Orientierung für die eigenen Selektionen setzt, kann die Relevanz einer zweiten Rationalität auch theoretisch eingeführt werden. Im Kontext der Ökonomisierung kann dies vor allem damit begründet werden, dass sich Geld als Medium kontextueller Steuerung, bei der ja auf die Regelung von Einzelheiten verzichtet werden kann, besonders eignet (vgl. Willke, 1998: 180ff.). Geld generalisiert die mit ihm verbundenen Wahlchancen in sachlicher Hinsicht, befreit sie also vom direkten Austausch von Gütern zwischen Käufern und Verkäufern, temporalisiert sie in zeitlicher Hinsicht, überlässt also die Bestimmungsmacht über die zeitliche Realisierung der Wahlchancen den Akteuren und macht in sozialer Hinsicht indifferent, abstrahiert also von der Ausrichtung der Zwecke und von der sozialen Position der Akteure (vgl. Willke, 1998: 200ff.). Die „Charakterlosigkeit“ des Geldes macht es anscheinend auch für das ansonsten selbststeuernde Mediensystem zum geeigneten Medium der kontextuellen Konditionierung.

2 Vgl. u.a. Rühl, 1980; Saxer, 1992; Marcinkowski, 1993; Blöbaum, 1994; Gerhards, 1994; Luhmann,1996a; Kohring, 1997; Medien Journal Themenheft „Systemtheorie der Medien“ 1/1997.

3 Weber (2000: 22f.) unterscheidet in seiner Studie ähnlich eine Gesellschaftsebene, eine Organisationsebene, eine Interaktionsebene und eine Textebene. 
Dabei wird gerade durch die Privatisierung und Deregulierung der Medien eine monetäre Kontextsteuerung gefördert, weil andere Rationalitäten zur Kontextsteuerung entfallen.

\subsection{Sachziel und ökonomisches Ziel von Medienorganisationen}

In den Medienorganisationen als struktureller Verfestigung des Mediensystems sind die Berührungs- und Interaktionsflächen zwischen Mediensystem und ökonomischem System besonders deutlich, haben doch Medienorganisationen sowohl ein Sachziel (Veröffentlichung) als auch ein ökonomisches Ziel (Geld). An der Etablierung des redaktionellen Managements und Marketings, vor allem aber des Medien-Controlling lässt sich nachvollziehen, dass die am Steuerungsmedium Geld orientierten Handlungen auch in Medienorganisationen tendenziell zunehmen.

Wird die Systemtheorie mit der Theorie rationalen Handelns verknüpft, dann können die jeweiligen ausdifferenzierten Teilsysteme als systemische „constraints“ von Akteurshandlungen konzipiert werden, die sowohl abstrakte substanzielle Ziele vorgeben als auch Mittel, um diese Ziele zu erreichen. „,Constraints' bezeichnen die strukturellen Restriktionen, unter denen Akteure ihre Wahlen, ihre ,choices' treffen und entsprechend handeln." (Gerhards, 1994: 80) Und dies so tun, dass sie ihre spezifischen Ziele mit möglichst geringem Aufwand erreichen. Je nachdem, welche generalisierte Handlungsorientierung (Veröffentlichung oder Geld) bzw. welche Entscheidungsprogramme (vgl. dazu auch Rühl, 1979) die spezifischen Handlungen der Akteure strukturieren, lassen sich in Medienorganisationen Abteilungen in solche, die in erster Linie dem ökonomischen System zuzurechnen sind, und in solche, die in erster Linie dem Mediensystem zuzurechnen sind, unterscheiden. Hier finden sich Anknüpfungspunkte an die medienökonomische Journalismusforschung, in der Programme als Zusammenfassung von Verfahren und Regeln einen Korridor festgelegter Strukturen bilden, der jedoch den Journalisten einen gewissen Handlungsspielraum einräumt (vgl. Altmeppen, 2000: 47f.).

Analytisch können also die Steuerungsmedien Geld und Publizität in Bezug auf ihre jeweilige Durchsetzungskraft als Handlungsorientierung im Organisationssystem untersucht werden. Ökonomisierung wird hier u.a. deutlich, weil sich der Beruf des Journalisten mittlerweile in Typen aufteilen lässt, die zu mehr oder weniger großen Teilen Managementtätigkeit ausüben, und die „Manager“-Journalisten zu gleichem Prozentsatz wie „Kern“-Journalisten in der oberen Redaktionshierarchie angesiedelt sind, also Durchsetzungsmacht haben (vgl. Weber, 2000: 117). Zugleich hat in der Einschätzung befragter Journalisten die Fremdsteuerung durch die Ökonomie auf verschiedenen Ebenen zugenommen (vgl. Weber, 2000: 146ff.).

\subsection{Interaktionen und Konvertierungsprozesse zwischen Medien und Ökonomie}

Damit ein wechselseitiger Leistungsaustausch zwischen Medien- und ökonomischem System stattfinden kann, müssen die gegenseitigen Leistungen permanent konvertiert werden. Für das ökonomische System ist der Transport von Werbebotschaften oder die Veröffentlichung von PR-Beiträgen, also die Ausstattung spezifischer, weil gewünschter Themen mit Publizität, eine spezifische Leistung, die das Mediensystem erbringt (Output) und die dann zum Input werden kann, wenn sie ökonomische Anschlusskommunikation im Sinne von Zahlungsverkehr konditioniert. Monetäre Einnahmen als konkreter Output ökonomischer Akte, z. B. aus dem Verkauf von Eigenproduktionen 
und Werbeschaltungen, werden zum Input beim Mediensystem über die Programmproduktion und damit über die Ausstattung von Themen mit Publizität. In der Beziehung zwischen Medien- und ökonomischem System muss also Geld in Publizität und Publizität in Geld übersetzt werden, damit die einzelnen Handlungen anschlussfähig sind. Dies entspricht auch der zunehmenden Vernetzung einzelner Subsysteme, die Beziehungen über die jeweiligen Systemgrenzen hinaus etablieren und Handeln über Systemgrenzen hinaus koordinieren und abstimmen müssen. Wie bei anderen Leistungsbeziehungen müssen dazu Institutionen etabliert werden, die die Konvertibilität unterschiedlicher Codes und Rationalitäten bewerkstelligen können (vgl. u. a. Münch, 1991: 284ff.; Willke 1996: 227f.).

Die Intensität dieser Konvertierungsprozesse und die Bemühungen, die in den reibungslosen Ablauf dieser Konvertierungen gesteckt werden, allen voran die Investitionen in die Medien-, Werbe- und Publikumsforschung, belegt die Relevanz der Beziehung zwischen Mediensystem und ökonomischem System und symbolisiert die Verquickung der beiden Systemrationalitäten (vgl. Siegert, 1993; Siegert, 1996). Dabei entspricht die Organisation dieser Forschung in Grundzügen dem, was systemtheoretisch als Verhandlungssystem bezeichnet wird. Mit der Institutionalisierung von Verhandlungssystemen werden sowohl die Kontextbedingungen für das Ganze generiert als auch die Teilsystemautonomien gestärkt (vgl. Willke 1996: 241ff.). Die Zusammensetzung der verschiedenen Kommissionen und Arbeitsgemeinschaften der empirischen Medien-, Werbe- und Publikumsforschung aus Akteuren des Mediensystems und des ökonomischen Systems verweist auf die Beibehaltung der Teilsystemautonomien, die Aushandlung von Forschungsrahmenbedingungen und Grenzwerten auf die gemeinsame Kontextsteuerung. Ergänzt wird sie mittlerweile durch das sich langsam etablierende Medien-Controlling (vgl. u. a. Beiträge im Sammelband Schneider/Knobloch 1999). Durch diese Institutionen wird einerseits Publizitätsleistung - Programmproduktion und -verbreitung - in Preise und Kosten umgerechnet, z. B. durch Kennzahlen des operativen Medien-Controlling. Andererseits werden Zahlungen in erwartbare Publizitätsleistungen konvertiert, indem z. B. mittels Tausender-Kontakt-Preise eingesetzte Gelder in Kontaktchancen übersetzt werden.

\section{Interpenetrationszonen von Medien und Ökonomie}

Die beispielhaften Ausführungen anhand der drei Ebenen, Selbst- und Kontextsteuerung, Sach- und ökonomisches Ziel sowie Interaktionen und Konvertierungsprozesse liefern zahlreiche Indizien für eine Ökonomisierung der Medien. Gleichwohl finden sich vielfältige Hinweise darauf, dass Publizität als Steuerungsmedium auch übergreifend an Relevanz gewinnt. Vor allem in der Diskussion um die Aufmerksamkeitsökonomie (vgl. u. a. Goldhaber, 1997; Franck, 1998) und in der Entwicklung der OnlineÖkonomie (vgl. u. a. European Communication Council, 1999; Latzer, 2000) zeigen sich Anhaltspunkte, die auf eine „Gleichberechtigung“ der Veröffentlichungsrationalität und damit des Mediensystems gegenüber der Geldrationalität und dem ökonomischen System hindeuten. Insgesamt verknüpfen sich ökonomische mit publizistischen Relevanzen und überlappen sich die beiden Rationalitäten Geld und Publizität derart, dass sich mehrere Bereiche ausmachen lassen, die sowohl der Logik des Mediensystems als auch der des ökonomischen Systems entsprechen. Auf diese Interpenetrationszonen soll anhand von drei Beispielen kurz eingegangen werden. 


\subsection{Interpenetrationszone Medieninhalte}

Auf dieser Ebene sind die beiden Systemrationalitäten gekoppelt, weil die Arbeitsweise des Mediensystems sowohl Selbstreferenz als auch Fremdreferenz sichert. Denn die Umweltwahrnehmung des Mediensystems konzentriert sich auf potenziell veröffentlichungswürdige und -fähige Themen und Personen bzw. reduziert sie auf ihren Veröffentlichungsaspekt hin, ist dabei aber prinzipiell offen gegenüber Themen aus allen Teilsystemen des gesellschaftlichen Systems. Dennoch deutet sich an, dass es eine stärkere Kopplung mit der Geld- als mit jeder anderen Systemrationalität gibt.

Besonders deutlich lässt sich dies am Beispiel PR und Werbung aufzeigen. PR und Werbung werden an dieser Stelle - auch aufgrund der Abgrenzung des Mediensystems - im Gegensatz zu Kohring/Hug (1997: 27ff.) als klassische Beispiele für Interpenetrationszonen auf der Inhalteebene angesehen, in denen sich die Systemlogiken Publizität und Geld vermischen (vgl. Westerbarkey 1995: 159ff.). Gleichwohl kann vor allem PR als Selbstbeobachtung im Gegensatz zu Journalismus als Fremdbeobachtung eingestuft werden. Ziel ist es, die ökonomisch orientierte PR-Eigenbeobachtung in journalistische Fremdbeobachtung zu überführen, indem die Aufbereitungs- und Darstellungsformen denen journalistischer Kommunikationsangebote (Nachrichtenwerte) angeglichen werden (vgl. Kohring/Hug 1997: 28).

Weitere, aktuelle Entwicklungen können als illustrative Ergänzungen herangezogen werden: Erstens die sich ausdifferenzierenden Werbesonderformen, wie Sponsoring oder Product Placement, und zweitens die zunehmende Hybridisierung, d. h. die Vermischung redaktioneller mit werblichen Inhalten, die sich auch in Begriffen wie „Advertorial“ niederschlägt (vgl. auch Weber, 2000: 23) sowie die symbiotische Verbindung zwischen Medien- und Warenwelt (vgl. auch Saxer, 1992: 98).

\subsection{Interpenetrationszone Online-Ökonomie}

In Arbeiten zur Online-Ökonomie wird - trotz des ökonomischen Duktus - diskutiert, ob Aufmerksamkeit als neue Währung zu verstehen ist (vgl. u. a. Goldhaber, 1997; Davenport, 2001), und ob sie als neue Restriktion in der Ökonomie angesehen werden muss, weil sie auch die Handlungspräferenzen ökonomischer Akteure strukturiert (vgl. auch Theis-Berglmair, 2000: 321ff.).

Beispielhaft zeigt sich dies an den in der Online-Ökonomie wichtigen Netzwerken: Einerseits wird die kritische Masse zu einem Schlüsselfaktor in der vernetzten Wirtschaft, was dazu führt, dass es Ziel von Online-Strategien sein muss, eine bestimmte Masse an Nutzern, letztlich also eine bestimmte Menge an Aufmerksamkeit, für ein Online-Angebot zu gewinnen. So kann es ökonomisch sinnvoll sein, seine Produkte und Leistungen zu verschenken, denn der Wert von Online-Netzwerken liegt weniger in den angebotenen Leistungen als vielmehr in seiner Größe, also im Umfang von Aufmerksamkeitszirkeln. Gleichzeitig basieren Online-Netzwerke auf Kommunikation und Publizität, was den Aspekt, welche Inhalte attraktiv sind und publiziert werden, in den Mittelpunkt rückt. Dabei erweisen sich gerade solche Strategien als erfolgreich, die mit „user generated content“ arbeiten, also einen fließenden Übergang zwischen Kommunikation und Publikation schaffen und die Veröffentlichung ,in die Hände der Nutzer legen“. Letztlich muss sich also das Management von Online-Netzwerken sowohl an Publizität als auch an Geld orientieren, um überhaupt erfolgreich sein zu können. 


\subsection{Mediatisierung der Ökonomie}

Dieser Trend lässt sich verallgemeinern, denn die Ökonomie und die ökonomisch orientierten Industrien werden zunehmend durch Akte bestimmt, die an der Veröffentlichungsrationalität orientiert sind: „In einer Zeit, in welcher der Markt durch ein riesiges Netzwerk der ökonomischen Kommunikation überspannt wird, sind Wechselwirkungen zwischen Kommunikations- und Wirtschaftskonjunktur an der Tagesordnung. Wie sich die Wirtschaftskonjunktur entwickelt, hängt davon ab, wie über die Konjunktur gesprochen wird.“ (Münch, 1991: 130)

In dieser Hinsicht lässt sich der Aufschwung der Organisations- und Unternehmenskommunikation als eine Art „Gegentrend“ zur Ökonomisierung der Medien sehen. Die bereits in der Politikwissenschaft und der politischen Kommunikation erarbeiteten Analysen zur wechselseitigen Instrumentalisierung von Medien und Politik lassen sich daher auf das Verhältnis von Medien und Ökonomie übertragen. Interpenetration muss in diesem Zusammenhang als wechselseitige Durchdringung der beiden Systeme Medien und Ökonomie mit den je anderen Leistungsanforderungen verstanden werden, die sich in der gegenseitigen Instrumentalisierung und damit verbunden in der Anpassung an die Operationslogik des anderen Systems äußert (vgl. Westerbarkey, 1995: 154ff.).

Während Medien gezwungen sind, mit „harten“ Kennzahlen - Erlösen, Kosten, monetären Bewertungen des Programmvermögens etc. - zu operieren und ökonomisch zu bestehen, sehen sich Unternehmen zunehmend mit den Mängeln ihrer öffentlichen Darstellung konfrontiert, die es mittels an die Handlungslogik der Medien angepasster PR zu beheben gilt. Dabei scheint die Börsennotierung von (Medien-)Unternehmen diese wechselseitige Durchdringung noch zu intensivieren, denn die Börsen-Performance ist nicht nur von den klassischen ökonomischen Ergebnissen und Entwicklungen abhängig, sondern auch und verstärkt von der öffentlichen Präsentation derselben und der begleitenden Kommunikationsarbeit.

\section{Fazit: Ökonomisierung der Medien und Mediatisierung der Ökonomie}

Verfolgt man die gesellschaftliche Entwicklung, treffen zwei Phänomene aufeinander: einerseits die Ökonomisierung, nicht nur des Mediensystems, sondern vieler gesellschaftlicher Bereiche, andererseits die steigende Bedeutung von Aufmerksamkeit, die erst durch Publizität in größerem Umfang generiert und gebündelt werden kann. Aufmerksamkeit scheint angesichts der Informationsfülle ein ebenso knappes Gut wie Geld zu sein. ${ }^{4}$ Es zeichnet sich nicht nur eine Ökonomisierung der Medien ab, sondern auch eine Mediatisierung der Ökonomie. Und beide Entwicklungen dehnen sich auf andere gesellschaftliche Teilsysteme aus, so dass wir letztlich von einer „ökonomisierten $\mathrm{Me-}$ dien- und Informationsgesellschaft" sprechen müssen. Dieser Entwicklung kann gerade mit systemtheoretischen Konzepten angemessen begegnet werden, weil sie die Unterscheidung von Systemrationalitäten und deren wechselseitige Durchdringung thematisieren.

4 Die Argumentation, Aufmerksamkeit wäre das knappe Gut schlechthin, kann nur mit einem sehr eingeschränkten Blickwinkel aufrechterhalten werden, denn sie ignoriert klassische Disparitäten. 


\section{Literatur}

Altmeppen, Klaus-Dieter (2000): Medienmanagement als Redaktions- und Produktionsmanagement. In: Karmasin, Matthias/Winter, Carsten (Hg.): Grundlagen des Medienmanagements. München, S. $41-58$.

Altmeppen, Klaus-Dieter (2001): Ökonomisierung und Medienunternehmen. In: Medien Journal, 25. Jg., Heft 1 (in Vorbereitung).

Blöbaum, Bernd (1994): Journalismus als soziales System. Geschichte, Ausdifferenzierung und Verselbständigung. Opladen.

Bruns, Thomas/Marcinkowski, Frank/Nieland, Jörg-Uwe/Ruhrmann, Georg/Schierl, Thomas (1996): Das analytische Modell. In: Schatz, Heribert (Hrsg.): Fernsehen als Objekt und Moment des sozialen Wandels: Faktoren und Folgen der aktuellen Veränderungen des Fernsehens. Opladen, S. 19 - 55.

Davenport, Thomas H. (2001): Attention Economy: Understanding the New Currency of Business. Havard (für Mai 2001 angekündigt).

European Communication Council (1999): Die Internet-Ökonomie. Strategien für die digitale Wirtschaft. Berlin/ Heidelberg/New York.

Franck, Georg (1998): Ökonomie der Aufmerksamkeit. Ein Entwurf. München/Wien.

Gerhards, Jürgen (1994): Politische Öffentlichkeit. Ein system- und akteurstheoretischer Bestimmungsversuch. In: Neidhardt, Friedhelm (Hg.): Öffentlichkeit, öffentliche Meinung, soziale Bewegungen. (Kölner Zeitschrift für Soziologie und Sozialpsychologie: Sonderheft 34). Opladen, S. $77-105$.

Goldhaber, Michael H. (1997): Attention Economy and the Net. (2 ${ }^{\text {nd }}$ Draft version of a talk presented at the conference on "Economics of Digital Information“. Cambridge, MA) In: http://www.well.com/user/mgoldh/AtEcandNet.html, letzter Zugriff 31.1.2001.

Kohring, Matthias (1997): Die Funktion des Wissenschaftsjournalismus. Ein systemtheoretischer Entwurf. Opladen.

Kohring, Matthias/Hug, Detlef (1997): Öffentlichkeit und Journalismus. Zur Notwendigkeit der Beobachtung gesellschaftlicher Interdependenz - ein systemtheoretischer Entwurf. In: Medien Journal, 21. Jg., Heft 1, S. 15 - 33.

Latzer, Michael (Hg.) (2000): Mediamatikpolitik für die Digitale Ökonomie. e-commerce, Qualifikation und Marktmacht in der Informationsgesellschaft. Innsbruck/Wien/München.

Luhmann, Niklas (1996a): Die Realität der Massenmedien. Opladen.

Luhmann, Niklas (1996b): Die Wirtschaft der Gesellschaft. Frankfurt/M.

Marcinkowski, Frank (1993): Publizistik als autopoietisches System. Politik und Massenmedien. Eine systemtheoretische Analyse. Opladen.

Medien Journal Themenheft „Systemtheorie der Medien“ (1997), 21. Jg., Heft 1.

Münch, Richard (1991): Dialektik der Kommunikationsgesellschaft. Frankfurt/M.

Rühl, Manfred (1979): Die Zeitungsredaktion als organisiertes soziales System. Bielefeld.

Rühl, Manfred (1980): Journalismus und Gesellschaft. Mainz.

Saxer, Ulrich (1992): Systemtheorie und Kommunikationswissenschaft. In: Burkart, Roland/Hömberg, Walter (Hg.): Kommunikationstheorien. Ein Textbuch zur Einführung. Wien, S. 91 110.

Schimank, Uwe (1985): Der mangelnde Akteursbezug systemtheoretischer Erklärungen gesellschaftlicher Differenzierung - Ein Diskussionsvorschlag. In: Zeitschrift für Soziologie, Jg. 14, S. $421-434$.

Schimank, Uwe (1988): Gesellschaftliche Teilsysteme als Akteursfiktionen. In: Kölner Zeitschrift für Soziologie und Sozialpsychologie, 40. Jg., S. $619-639$.

Schmidt, Siegfried J. (1996): Die Welten der Medien. Grundlagen und Perspektiven der Medienbeobachtung. Braunschweig-Wiesbaden.

Schneider, Beate/Knobloch, Silvia (Hg.) (1999): Controlling-Praxis in Medien-Unternehmen. Neuwied/Kriftel.

Siegert, Gabriele (1993): Marktmacht Medienforschung. Die Bedeutung der empirischen Medienund Publikumsforschung im Medienwettbewerbssystem. München. 
Siegert, Gabriele (1996): Die Beziehung zwischen Medien und Ökonomie als systemtheoretisches Problem. In: Mast, Claudia (Hg.). Markt - Macht - Medien. Publizistik zwischen gesellschaftlicher Verantwortung und ökonomischen Zielen. Konstanz, S. 43 - 55.

Theis-Berglmair (2000): Aufmerksamkeit und Geld, schenken und zahlen. Zum Verhältnis von Publizistik und Wirtschaft in einer Kommunikationsgesellschaft - Konsequenzen für die Medienökonomie. In: Publizistik, 45. Jg., S. 310 - 329.

Weber, Stefan (2000): Was steuert Journalismus? Ein System zwischen Selbstreferenz und Fremdsteuerung. Konstanz.

Weischenberg, Siegfried (1994): Journalismus als soziales System. In: Merten, Klaus/Schmidt, Siegfried J./Weischenberg, Siegfried (Hg.): Die Wirklichkeit der Medien. Eine Einführung in die Kommunikationswissenschaft. Opladen, S. $427-454$.

Westerbarkey, Joachim (1995): Journalismus und Öffentlichkeit. Aspekte publizistischer Interdependenz und Interpenetration. In: Publizistik, 40. Jg., S. $152-162$.

Willke, Helmut (1996): Systemtheorie 1. Grundlagen: Eine Einführung in die Grundprobleme der Theorie sozialer Systeme. Stuttgart.

Willke, Helmut (1998): Systemtheorie 3. Steuerungstheorie: Grundzüge einer Theorie der Steuerung komplexer Sozialsysteme. Stuttgart. 